\title{
Monotherapy or Polytherapy for Epilepsy?
}

\author{
Alan Guberman
}

\begin{abstract}
Monotherapy has been promoted as the ideal in epilepsy treatment because of reduced side effects, absence of drug interactions, better compliance, lower cost and, in many cases, improved seizure control compared to polytherapy. The question of monotherapy vs. polytherapy has assumed increasing importance with the availability of multiple new antiepileptic drugs (AEDs), initially tested as add-on agents. The new drugs clobazam, lamotrigine, vigabatrin, gabapentin and topiramate, have also been shown to be effective as monotherapy. These data bring up the possibility of using them as first-line agents. However, a high percentage of patients with resistant epilepsy are treated with polytherapy, which probably benefits only a minority of them. The availability of multiple drugs with different mechanisms of action favours the possibility of "rational polytherapy", taking advantage of possible synergism, a yet unproven concept. This article reviews the theoretical advantages of monotherapy and monotherapy with traditional and newer AEDs.
\end{abstract}

\begin{abstract}
RÉSUMÉ: Monothérapie ou polythérapie dans le traitement de l'épilepsie? La monothérapie a été vantée comme étant le traitement idéal de l'épilepsie parce qu'elle provoque moins d'effets secondaires, qu'il n'y a pas d'interactions médicamenteuses, qu'il existe une meilleure fidélité au traitement, un coût moindre et, dans bien des cas, une amélioration du contrôle des crises par rapport à la polythérapie. La question de la monothérapie vs. la polythérapie a pris une importance croissante depuis l'avènement de plusieurs nouveaux médicaments antiépileptiques évalués initialement comme traitement d'appoint dans l'épilepsie. On a maintenant démontré que le clobazam, la lamotrigine, la vigabatrine, la gabapentine et le topiramate, de nouveaux médicaments, sont également efficaces en monothérapie. Ces données soulèvent la possibilité de les utiliser comme agents de première ligne chez certains patients. Cependant un fort pourcentage de patients qui ont une épilepsie résistante au traitement sont traités par une polythérapie, mais probablement qu'une minorité de ces patients seulement en bénéficie. La disponibilité de plusieurs médicaments qui ont des mécanismes d'action différents offre la possibilité d'une "polythérapie rationnelle", permettant de tirer profit de synergismes possibles, un concept qui n'a pas été prouvé à date. Dans cet article, nous revoyons les avantages théoriques de la monothérapie et les données sur la monothérapie en ce qui a trait aux médicaments antiépileptiques traditionnels et récents.
\end{abstract}

Can. J. Neurol. Sci. 1998; 25: S3-S8

The treatment for most types of epilepsy, for many years, was a combination of phenytoin and phenobarbital, even as initial treatment. The concept behind this was that these drugs had a synergistic effect and that side effects could be minimized by using two drugs with a favourable pharmacodynamic interaction at relatively low doses. Polytherapy was the standard in epilepsy until relatively recently when it became apparent that antiepileptic drugs were effective as monotherapy and that adverse effects and drug interactions could be avoided or reduced with single drug treatment. Monotherapy has become the goal in epilepsy in the past 10-15 years. This change in approach reflects increasing attention to quality of life issues in epilepsy.

Early studies showed that in many cases of poorly-controlled epilepsy, simplification of antiepileptic drug (AED) regimes could actually lead to an improvement in seizure control. ${ }^{1-3}$ Subsequent studies, with the U.S. VA study as the prototype, compared monotherapy treatment with standard drugs in newly-diagnosed patients and showed that polytherapy had very little additional benefit in patients who had failed monotherapy with two first-line agents. ${ }^{4}$ However, with the advent of a host of new AEDs, initially tested in an add-on context with intractable patients, the issue of monotherapy vs. polytherapy is once again assuming importance. Only recently are data available that allow us to recommend the use of the new agents as monotherapy. The relative merits of monotherapy and polytherapy in a given patient are not always easy to determine and more data are required to permit a scientifically-based choice between these approaches. The order of selection of agents for polytherapy is still to be determined.

\section{Advantages of monotherapy}

Around 1980 the longstanding practice of using polytherapy for epilepsy was systematically challenged. Reynolds and

\footnotetext{
From the Division of Neurology, Ottawa Hospital, General Campus, 501 Smyth Road, Ottawa

RECEIVED JUNE 30, 1998.

Reprint requests to: Alan Guberman, Division of Neurology, Ottawa Hospital, General Campus, 501 Smyth Road, Ottawa, Ontario, Canada K1H 8L6
} 
Table 1: Potential Advantages of Monotherapy.

No drug interactions with other AEDs

Fewer adverse effects (including teratogenicity)

Improved compliance

Cheaper

Better seizure control (when polytherapy reduced)

Improved quality of life.

Shorvon ${ }^{5}$ outlined the reasons for the popularity of polytherapy in epilepsy including: the intractability and chronicity of epilepsy in many patients, combination drug formulations (e.g., phenytoin and phenobarbital), established traditional practices of adding-on successive drugs and the lack of adequate monotherapy AED trials. The routine use of polytherapy was questioned as new knowledge became available on drug interactions. For example, it was frequently observed that it was difficult to obtain therapeutic serum levels of valproate in patients taking carbamazepine concurrently. ${ }^{6}$ The teratogenicity of drugs such as valproate became apparent when they were used in combination with inducing agents. The enhanced teratogenesis has been attributed to the formation of higher concentrations of teratogenic metabolites. ${ }^{7}$ However, the most compelling argument against polytherapy is the potential for chronic additive toxicity resulting from the use of multiple agents. The advantages of monotherapy over polytherapy are outlined in Table 1.

In the late $1970 \mathrm{~s}$, two agents, effective as monotherapy, were emerging as the drugs of choice for most types of epilepsy: valproic acid and carbamazepine. In a retrospective review of the impact of adding a second AED to patients taking a variety of AEDs in monotherapy, Shorvon and Reynolds ${ }^{8}$ found an improvement of seizures $(\geq 50 \%)$ in only $38 \%$ of patients.

Studies have examined the effects of discontinuing drugs to achieve monotherapy in patients on multiple AEDs. ${ }^{1-3}$ Shorvon and Reynolds were able to reduce drugs cautiously in 40 adult patients on long-term polytherapy to reach monotherapy successfully in $72 \%$ of cases. ${ }^{1}$ In $55 \%$ of patients, seizures actually improved ( $\geq 50 \%$ reduction) while in only $17 \%$ did seizures increase with monotherapy. Alertness, concentration, energy, mood and sociability were markedly improved. Schmidt reported similar success in reduction of two-drug therapy to monotherapy in 36 patients. ${ }^{2}$ Thirty-six percent of patients had improvement in seizure control and only $17 \%$ deteriorated. Theodore and Porter showed that discontinuation of sedative AEDs actually resulted in improvement in seizure control as well as increased alertness. ${ }^{3}$ The reasons for improved seizure control with simplification to monotherapy are unclear; possibilities include a reduction of drug interactions allowing the achievement of therapeutic levels of the remaining drug, improved compliance, elimination of toxic effects which exacerbate seizures or a combination of these factors.

The VA cooperative study ${ }^{4}$ was the first well-controlled prospective design to show the potential of monotherapy in newly-diagnosed partial epilepsy in adults. Forty-seven percent of patients had all seizures completely controlled on carbamazepine for 12 months. Phenytoin, primidone and phenobarbital performed almost as well. Addition of a second drug in those failing monotherapy led to complete seizure control in only an additional $11 \%$ but $40 \%$ did have a reduction in seizures. Side effects, however, were more prevalent on two drugs.

The major impetus to maintain or achieve monotherapy is reduction of the additive neurotoxic, including cognitive, side effects. A pediatric study, for example, demonstrated the overall incidence of side effects on one, two or three drug therapy was $22 \%, 34 \%$ and $44 \%$ respectively. ${ }^{9}$ Several studies have examined cognitive and other neurotoxic effects of AEDs, and have shown that simplification to monotherapy has produced a reduction in these side effects. Certain idiosyncratic reactions, such as the rare fatal hepatotoxicity with valproate are reduced with monotherapy. ${ }^{10}$ Rash with lamotrigine is another example of an idiosyncratic reaction with a lower incidence with monotherapy. ${ }^{11}$ The incidence of rash is increased when lamotrigine is given to patients receiving valproate, presumably caused by the inhibition of LTG metabolism by valproate.

Lammers et al. ${ }^{12}$ used an original approach to compare retrospectively the incidence of neurological adverse events in two groups of epileptic patients in Holland - one treated with monotherapy and the other with polytherapy. The groups were stratified according to the ratio of prescribed daily dose to the assumed average adult effective daily dose. There was no difference between monotherapy and polytherapy patients in the incidence of neurological side effects after the adjustments for dose. In other words, it was the total drug dosage rather than poly or monotherapy that was the most important determinant of the neurological side effects after the adjustments for dose. This study was weakened by the fact that blood levels were not used, patients were not randomized to either poly or monotherapy, and specific drugs were not matched in the two groups. The same investigators have recently published a similar analysis based on a literature review of 15 published clinical trials. ${ }^{13}$ An attempt was made to determine whether total drug load or number of drugs (polytherapy) was better correlated with numbers of adverse effects (neurotoxicity). Toxicity on polytherapy appeared to relate better to total drug load than to number of drugs administered.

Experimental evidence exists suggesting that the combination of similarly-acting agents such as phenytoin and carbamazepine does not lead to a different therapeutic index than if the agents are used alone. Morris et al. ${ }^{14}$ studied phenytoin and carbamazepine alone and in combination in the MES model in mice and found no justification for using these agents in combination.

\section{Monotherapy studies with valproate (VPA)}

Valproate was one of the first AEDs to be promoted for use in monotherapy. An early open monotherapy study with VPA showed that it was effective in controlling all primary generalized seizure types in $83 \%$ of 118 patients (children and adults), some of whom had failed previous therapy. ${ }^{15}$ There have been three large comparative randomized monotherapy trials involving VPA in newly-diagnosed epilepsy in adults. The second VA study, comparing VPA to carbamazepine (CBZ) in 480 treated and untreated patients with partial epilepsy in a double-blind design, ${ }^{16}$ the EPITEG study comparing VPA to CBZ in 300 patients with partial or primary generalized epilepsy in an open design ${ }^{17}$ and the MRC study included 243 patients who were randomized in an open design to one of 4 drugs: phenobarbitone, phenytoin, carbamazepine or sodium valproate. ${ }^{18}$ The 
EPITEG study found that VPA and CBZ controlled both generalized tonic-clonic and partial seizures equally well over 3 years and that withdrawals for adverse events were $15 \%$ in the CBZ group vs. $5 \%$ in the VPA group. In the MRC study, doses were adjusted according to clinical response and plasma levels. The follow-up time was approximately 100 months in the MRC study compared to 24 months in the VA study. The VA study used slightly higher blood levels $(<150 \mu \mathrm{g} / \mathrm{ml}$ vs. $<100 \mu \mathrm{g} / \mathrm{ml})$ than the MRC study. The MRC study found no difference between remission rates achieved at the end of 3 years, or retention rates when comparing VPA and CBZ. Discontinuations from $C B Z$ tended to occur early and were related to rash or somnolence whereas they tended to occur later with VPA due to weight gain. The VA study showed no difference between retention rates (an indicator of global outcome) with VPA or CBZ at 12 months $(N=150)$. The efficacy for secondarily generalized tonic-clonic seizures did not differ between the two drugs but $\mathrm{CBZ}$ was somewhat more effective in controlling partial seizures. These studies cannot be readily compared because of differences in patient populations and methodologies. However it is clear that VPA does have efficacy in partial seizures although possibly slightly less than CBZ, especially in patients with higher pre-treatment seizure frequency. ${ }^{19}$ A U.K. pediatric study $(\mathrm{N}=263$ ) comparing VPA and CBZ showed comparable efficacy between the two drugs. ${ }^{20}$

Another randomized open study comparing VPA, CBZ, PHT and $\mathrm{PB}$ monotherapy in newly-diagnosed childhood partial epilepsy showed little difference in efficacy among the four drugs. Seventy-three percent of children overall achieved a oneyear remission by 3 years. ${ }^{21}$

A recent double-blind concentration-response add-on and withdrawal to monotherapy trial of divalproex sodium (VPA) in poorly-controlled partial epilepsy has been reported. ${ }^{22}$ This study demonstrated the efficacy of valproate used in monotherapy for partial epilepsy. One hundred and thirty-four patients, 10 years of age or older with partial epilepsy, were randomized to the low VPA plasma level group $(175-345 \mu \mathrm{mol} / \mathrm{L})$ and 131 to the high concentration group $(550-1040 \mu \mathrm{mol} / \mathrm{L})$. Phenytoin and carbamazepine were the most common baseline AEDs which were gradually withdrawn after VPA was added. There was a 16-week dosage maintenance phase in patients who achieved monotherapy (96/134 and $47 / 131$ in the high and low concentration groups respectively). Median reduction in seizure frequency (complex partial and secondarily generalized tonic-clonic) was $30 \%$ and $70 \%$ respectively vs. baseline in the high concentration group which was significantly greater $(p=0.001)$ than in the low concentration group (median increase of $19 \%$ in complex partial and $22 \%$ in secondarily generalized seizures). Responder rates ( $\geq 50 \%$ seizure reduction) were $38 \%$ in the high concentration group vs. $23 \%$ in the low concentration group. Nine percent of patients were seizure-free in the high concentration group vs. $2 \%$ in the low concentration group.

Christie et al. ${ }^{23}$ reported a double-blind randomized, controlled trial comparing oxcarbazepine (OXCBZ) to VPA in newly-diagnosed adult partial $(\mathrm{N}=154)$ or generalized tonicclonic $(N=95)$ epilepsy. Observations were made over a 48 week maintenance period with a mean $O X C B Z$ and VPA daily dose of $1053 \mathrm{mg}$ and $1146 \mathrm{mg}$ respectively. The two drugs were equivalent by virtually all measures of efficacy and tolerability. Fifty-seven percent of the OXCBZ and $54 \%$ of the VPA patients were seizure-free over 12 months. These studies have shown valproate to be effective when used as monotherapy for partial epilepsy in newly-diagnosed patients as well as in poorly-controlled patients.

\section{Monotherapy studies with the new antiepileptic drugs}

The new AEDs have been tested more recently with a variety of monotherapy designs since, similarly to the traditional drugs, they would be expected to be effective when given alone. The designs which have been used include: comparison to an established drug in newly-diagnosed patients; add-on to ongoing therapy and then withdrawal to monotherapy and short-term comparison to an "active" control or placebo. The last design has been used in hospitalized patients undergoing drug withdrawal as part of a pre-surgical workup. The following is a brief review of the monotherapy data for the new AEDs available in Canada.

\section{Lamotrigine (LTG)}

Six major lamotrigine monotherapy studies of various designs in adults have been reported. ${ }^{24-29}$ An open multicentre European trial comparing lamotrigine $100 \mathrm{mg}$ or $200 \mathrm{mg}$ and carbamazepine $600 \mathrm{mg}$ in newly-diagnosed or recurrent adult epilepsy showed that lamotrigine was equally effective to carbamazepine but LTG was better tolerated. Marginally more patients $(5.1 \%)$ were withdrawn in the CBZ group than in the LTG group (4.4\%). . $^{24}$

A large double-blind randomized European multicentre study compared LTG to CBZ in patients over 12 years old with newlydiagnosed partial epilepsy or generalized tonic-clonic seizures. ${ }^{25}$ Median dose in patients receiving LTG $(\mathrm{N}=131)$ was 150 $\mathrm{mg} /$ day and $600 \mathrm{mg}$ in the CBZ group $(\mathrm{N}=129)$. Efficacy, measured as time to first seizure after 6 weeks' treatment, did not differ between treatment groups; the proportion of patients seizure-free (all seizures) during the last 40 weeks of treatment were $26 \%$ for LTG and $29 \%$ for CBZ. Sixty-five percent of the LTG patients vs. $51 \%$ of the CBZ group completed the study (significant at $p=0.018$ ) and the difference was due to more adverse events on CBZ. Nineteen percent of patients in each group developed rash but only 12 patients in the LTG group vs. 17 in the CBZ group were withdrawn due to rash. Blood levels were not measured and relatively low doses were used. Efficacy differences between groups may have been more apparent at higher doses which is suggested by the low rate of complete seizure control in either group.

A similar study comparing LTG to phenytoin (PHT) was reported by Steiner et al. ${ }^{26}$ Again LTG was as effective as PHT and had fewer adverse events.

Faught reported on U.S. patients completing U.S. doubleblind add-on trials of LTG. ${ }^{27}$ Discontinuation to LTG monotherapy in 69 patients was attempted; $72 \%$ of patients were successfully converted, $30 \%$ of whom were seizure-free for up to 4 years.

A European LTG add-on study followed by discontinuation to LTG monotherapy and then observation for 12 weeks involved 338 intractable patients. ${ }^{28}$ Fifty-six percent of patients successfully achieved monotherapy, eighteen percent completed the trial on LTG monotherapy and $19 \%$ were free of all seizures. Withdrawal due to skin rash was seen in $38 \%$ of patients in whom LTG was added to VPA and rapid dose escalation was 
used vs. $8 \%$ on VPA with slow dose escalation and $0-4 \%$ on $\mathrm{CBZ}$ or PHT independently of dose-escalation. Brodie et al." have recently re-analyzed the same data with an additional 9 patients who were receiving phenobarbital as the baseline drug. They reported that $23 \%$ of patients achieved LTG monotherapy, idiopathic generalized tonic-clonic seizures showed a responder rate of $61 \%$ vs. $43 \%$ in partial seizures and, independently of seizure type, LTG added to VPA seemed to result in a significantly greater response rate.

The most recent study compared add-on LTG (up to 500 $\mathrm{mg} /$ day) to VPA (1000 mg/day) followed by discontinuation to monotherapy for 12 weeks in adults. ${ }^{29}$ Only forty-four percent in the LTG group ( $\mathrm{N}=50)$ met escape criteria vs. $80 \%$ in the VPA group $(\mathrm{N}=64)$. The relatively low doses of VPA should be noted. Two patients in the LTG group developed Stevens-Johnson syndrome.

\section{Clobazam (CLB)}

Two early open-label pediatric trials suggested that clobazam was effective in monotherapy. ${ }^{30,31} \mathrm{~A}$ well-designed, controlled double-blind, double-dummy monotherapy comparative trial with clobazam in pediatric epilepsy has recently been reported by Camfield. ${ }^{32}$ Fifteen Canadian centres entered 235 patients, aged 6 months -18 years, with partial epilepsy ( $89 \%$ of cases) or primary generalized epilepsy with tonic-clonic seizures $(11 \%)$. The patients were either drug-naive $(n=115)$ or had failed treatment on 1 drug $(n=120)$. Overall, 119 patients were randomized to clobazam, 78 to carbamazepine and 38 to phenytoin. Equivalent dosing was used and doses were adjusted according to levels. Fifty-five percent of patients were retained on clobazam for 12 months vs. $57 \%$ on the other two drugs. There were no significant differences between clobazam and the other drugs in terms of side effects. Interestingly, tolerance, strictly defined, was not greater with clobazam than the other drugs. This study has shown that clobazam is as effective and well tolerated as PHT and CBZ when used in monotherapy in this group of pediatric patients with relatively mild epilepsy.

\section{Vigabatrin (VGB)}

Fisher et al. (1996) recently reviewed monotherapy studies with vigabatrin. ${ }^{33}$ In 15 studies representing 300 patients, $2 / 3$ of patients were improved and $40 \%$ were seizure-free. There have been 3 major monotherapy comparative studies. The first study was an open-label study in newly-diagnosed adult cases of partial epilepsy randomized either to vigabatrin $(n=50)$ or carbamazepine. ${ }^{34}$ Although $26 \%$ of the VGB group dropped out due to lack of efficacy vs. $6 \%$ in the CBZ group, $24 \%$ of the CBZ group dropped out due to adverse events vs. 0 in the VGB group. The efficacy of the two drugs was similar in patients with complex partial seizures without secondary generalization.

Tangenelli and Regestra randomized 51 newly-diagnosed cases of partial epilepsy to either carbamazepine or vigabatrin. ${ }^{35}$ Failures were crossed over to the other drug and non-responders to monotherapy with either drug were treated with a combination of the two drugs. There was no significant difference in efficacy between the two drugs: $17 / 37$ patients had complete control of seizures on VGB and $20 / 39$ on CBZ. Side effects were more frequent and severe on CBZ. Brodie has recently reported the results of a multicentre double-blind study comparing vigabatrin to valproate as monotherapy in patients with par- tial seizures who had been resistant to carbamazepine. ${ }^{36}$ VPA or VGB was added to CBZ; CBZ was then discontinued to achieve monotherapy. Thirty-six percent of patients on VGB achieved monotherapy and $42 \%$ of patients on valproate. Twenty-six percent of patients on VGB vs. $29 \%$ on VPA were seizure-free at the end of the study. One patient had "confusion" and 1 patient psychosis as serious events on VGB but side effects were otherwise quite comparable. Vigabatrin appears to be as effective as valproate when used as second line monotherapy, but has greater potential toxicity (including recently recognized retinal toxicity in some patients with long-term use) ${ }^{37}$

\section{Gabapentin (GBP)}

Gabapentin monotherapy has been examined in three adult studies. ${ }^{38-40}$

A multicentre 8-day "active control" double-blind randomized study was conducted on 82 in-patients in whom antiepileptic drugs were discontinued for presurgical EEG monitoring. Gabapentin doses of $3600 \mathrm{mg} / \mathrm{day}$ and $300 \mathrm{mg} / \mathrm{day}$, achieved within 24 hours, were compared. The higher dose was significantly more effective, as judged by time to the fourth seizure and the number of patients completing 8 days without experiencing 4 seizures. The high dose was also well-tolerated. ${ }^{38}$ This study established efficacy of GBP in short-term monotherapy.

A double-blind add-on and discontinuation to monotherapy study in refractory out-patients with partial epilepsy was done at several U.S. centres. ${ }^{39}$ Two hundred and seventy-five refractory patients, 23 years of age or older, received gabapentin at doses of either $600 \mathrm{mg}(\mathrm{n}=93), 1200 \mathrm{mg}(\mathrm{n}=90)$ or $2400 \mathrm{mg}(\mathrm{n}=$ 91) per day. The study was conducted as add-on therapy over 10 weeks (including an 8-week taper period), followed by monotherapy for 16 weeks. Exit criteria indicating worsening were strictly defined. Time to exit, mean time on monotherapy and completion rates were equivalent in the three groups (completion ranged from $25-31 \%$ ). Only 7 patients withdrew due to adverse events. The low rate of completion and lack of a doseresponse relationship suggest that gabapentin was not very effective in most of these intractable patients. However an increase in seizures during withdrawal of carbamazepine as compared to the other 2 drugs may have partially obscured the efficacy of gabapentin. Also relatively low doses were used.

An international study randomized 292 newly-diagnosed untreated patients with partial epilepsy to one of four parallel groups: GBP $300 \mathrm{mg}$, $900 \mathrm{mg}$ or $1800 \mathrm{mg}$ per day or carbamazepine $600 \mathrm{mg} /$ day. ${ }^{40}$ Gabapentin was administered in a double-blind fashion and carbamazepine in an open fashion. Patients on the two higher doses of GBP achieved retention significantly longer than those on $300 \mathrm{mg} /$ day. Completion rates for GBP at the two higher doses were $39 \%$ and $38 \%$ respectively, quite similar to the completion rate of $37 \%$ on carbamazepine. Withdrawal for adverse events was $24 \%$ for carbamazepine versus $14 \%$ for GBP $1400 \mathrm{mg} /$ day. Gabapentin compared favourably to carbamazepine over 6 months in this group of newly-diagnosed patients. It appears that gabapentin can be effective as monotherapy, particularly in patients with mild epilepsy, and is better tolerated than carbamazepine.

\section{Topiramate (TPM)}

Topiramate (TPM) is the newest AED released in Canada and only a minimal amount of monotherapy data is available. 
Forty-eight patients with partial epilepsy were titrated to either topiramate $100 \mathrm{mg}$ or $1000 \mathrm{mg}$ in a double-blind add-on to discontinuation study. ${ }^{41}$ Therapeutic success as measured by completion of the conversion and monotherapy periods and by fewer patients meeting exit criteria significantly favoured the highdose group. Twenty-five percent of the high-dose TPM group experienced $>75 \%$ seizure reduction (13\% complete control) as opposed to $8 \%$ ( $0 \%$ complete control) in the low-dose group. No major differences in adverse events were noted between the 2 groups and TPM was tolerated adequately or well in $88 \%$ of the high-dose group and $96 \%$ of the low-dose group.

\section{Polytherapy: rational or otherwise}

Although monotherapy in treating epilepsy is a desirable goal, some patients with intractable forms of epilepsy, such as those with the Lennox-Gastaut syndrome, may require and benefit from polytherapy. The argument for polytherapy is based in some cases on multiple seizure types requiring drugs with different mechanisms of action. In other cases, synergy may be operating or two drugs may have additive efficacy but not additive toxicity. However, drugs are often added without regard to rational combinations or to whether some of the pre-existing drugs could be discontinued. The concept of polytherapy in epilepsy needs to be re-evaluated because of the availability of 5 new agents (clobazam, vigabatrin, gabapentin, lamotrigine, topiramate) in the last 6 years. The question is: "Are there rational combinations of AEDs which can, on the basis of useful pharmacodynamic synergisms or pharmacokinetic interactions without intolerable neurotoxicity, offer advantages over high doses of single agents?" Without much available data or a consistent definition, we are increasingly seeing "rational polytherapy" promoted. The term usually refers to combining agents with different, presumably synergistic, mechanisms of action. An example would be combining a sodium channel blocker (e.g., carbamazepine) with a GABA-enhancing agent (e.g., vigabatrin). Although this approach is theoretically appealing, there is very little clinical or laboratory evidence to support it. Many "irrational" combinations are effective as is frequently witnessed during add-on clinical trials.

Rational combinations can also be theoretically recommended on pharmacokinetic grounds. For example, the addition of clobazam to an inducing agent such as carbamazepine results in higher levels of the $\mathrm{N}$-desmethylclobazam metabolite, with potential increased therapeutic benefits.

We are still lacking studies comparing the newer agents with one another, both in polytherapy and in monotherapy. Hopefully, as we better understand the underlying pathophysiology of epilepsy and have more data on the use of specific drug combinations, we will be able to use polytherapy with the new drugs, when necessary, in a more rational manner. Studies quoted above confirm that the newer AEDs are effective to a greater or lesser degree when used as monotherapy and are often better tolerated than the traditional agents. In the future cost may prove to be the main barrier to using these drugs as first-line agents.

\section{REFERENCES}

1. Shorvon SD, Reynolds EH. Reduction in polypharmacy for epilepsy. Brit Med J 1979; 2: 1023-1025.

2. Schmidt D. Reduction of two drug therapy in intractable epilepsy. Epilepsia 1983; 24: 368-376.
3. Theodore WH, Porter RJ. Removal of sedative-hypnotic antiepileptics from the regimens of patients with intractable epilepsy. Ann Neurol 1983; 13: 320-324.

4. Mattson RH, Cramer JA, Collins JF, et al. Comparison of carbamazepine, phenobarbital, phenytoin and primidone in partial and secondarily generalized tonic-clonic seizures. $N$ Engl J Med 1985; 313: 145-151.

5. Reynolds EH, Shorvon SD. Monotherapy or polytherapy for epilepsy. Epilepsia 1981; 22: 1-10.

6. Bourgeois BFD. Pharmacologic interactions between valproate and other drugs. Am J Med M88; 84 (Suppl 1A): 29-33.

7. Lindout D. Pharmacogenetics and drug interactions: role in antiepileptic drug-induced teratogenesis. Neurology 1992; 42 (Suppl 5): 43-47.

8. Shorvon SD, Reynolds EH. Unnecessary polypharmacy for epilepsy. Brit Med J 1977; 1: 1635-1637.

9. Beghi E, DeMascio R, Sasanelli $F$ et al. Adverse reactions to antiepileptic drugs: a multicentre survey or clinical practice. Epilepsia 1986; 27: 323-330.

10. Bryant AE III, Dreifuss FE. Valproic acid hepatic fatalities. III. U.S. experience since 1986. Neurology 1996; 46: 465-469.

11. Brodie MJ, Yven AWC. Lamotrigine substitution study: evidence for synergism with sodium valproate? Epilepsy Res 1997; 26: 423-432.

12. Lammers MW, Hekster YA, Keyser A, et al. Monotherapy or polytherapy for epilepsy revisited: a quantitative assessment. Epilepsia 1995 ; 36: 440-446.

13. Deckers CLP, Hekster YA, Keyser A, et al. Reappraisal of polytherapy in epilepsy: a critical review of drug load and adverse effects. Epilepsia 1997; 38: 570-575.

14. Morris JC, Dodson E, Hatlelid M, et al. Phenytoin and carbamazepine alone and in combination: anticonvulsant and neurotoxic effects. Neurology 1987; 37: 1111-1118.

15. Bourgeois B, Beaumanoir A, Blajev B et al. Monotherapy with valproate in primary generalized epilepsies. Epilepsia 1987; 28 (Suppl 2): S8-S11.

16. Mattson RH, Cramer JA, Collins JF, et al. A comparison of valproate with carbamazepine for the treatment of complex partial seizures and secondarily generalized tonic-clonic seizures in adults. N Engl J Med 1992, 327: 765-771.

17. Richens A, Davidson DLW, Cartlidge NF, et al. A multicentre comparative trial of sodium valproate and carbamazepine in adult onset epilepsy. J Neurol Neurosurg Psychiatry 1994; 37: 682687.

18. Heller AJ, Chesterman P, Elwes RDC, et al. Phenobarbitone, phenytoin, carbamazepine, or sodium valproate for newly diagnosed adult epilepsy: a randomized comparative monotherapy trial. J Neurol Neurosurg Psychiatry 1995; 58: 44-50.

19. Seino M. A comment on the efficacy of valproate in the treatment of partial seizures. Epilepsia 1994; 35 (Suppl 5): S101-S104.

20. Verity CM, Hosking G, Easter DJ. A multicentre comparative trails of sodium valproate and carbamazepine in pediatric epilepsy. Develop Med Child Neurol 1995; 37: 97-108.

21. De Silva M, MacArdle B, McGowan M, et al. Randomized comparative monotherapy trial of phenobarbitone, phenytoin, carbamazepine, or sodium valproate for newly-diagnosed childhood epilepsy. Lancet 1996; 347: 709-713.

22. Beydoun A, et al. Safety and efficacy of divalproex sodium monotherapy in partial epilepsy: a double-blind, concentration response design clinical trial. Neurology 1997; 48: 182-188.

23. Christie W, Krämer G, Vigonius V, et al. A double-blind controlled clinical trial: oxcarbazepine versus sodium valproate in adults with newly diagnosed epilepsy. Epilepsy Res 1997; 45!-460.

24. Reunanen M, Dam M, Yven AWC. A randomized open multicentre comparative trial of lamotrigine and carbamazepine as monotherapy in patients with newly diagnosed or recurrent epilepsy. Epilepsy Res 1996; 23: 149-155.

25. Brodie MJ, Richens A, Yven AWC, et al. Double-blind comparison of lamotrigine and carbamazepine in newly-diagnosed epilepsy. Lancet 1995 ; 345: 476-479.

26. Steiner TJ, Yven AWC. Comparison of lamotrigine and phenytoin monotherapy in newly diagnosed epilepsy. Epilepsia 1994; 35 (Suppl 8): S31. 
27. Faught E. Lamotrigine monotherapy in patients with refractory partial-onset seizures. In: Loiseau P, ed. Lamotrigine-A Brighter Future. International Congress and Symposium Series \#214. London: Royal Society of Medicine Press. 1996: 37-42.

28. Perucca E. Add-on trial of lamotrigine followed by withdrawal of concomitant medication and stabilization on monotherapy. In: Loiseau P, ed. Lamotrigine-A Brighter Future. International Congress and Symposium Series \#214. London: Royal Society of Medicine Press. 1996: pp. 23-30.

29. Chang G, Vazquez B, Gilliam F, et al. Lamictal (lamotrigine) monotherapy as an effective treatment for partial seizures. Neurology 1997; 48 (Suppl): A335.

30. Dulac O, Figueroa D, Rey E, et al. Monothérapie par le clobazam dans l'épilepsie de l'enfant. Presse Méd 1983; 12: 1067-1069.

31. Plouin P, Jalin C. EEG changes in epileptic children treated with clobazam as monotherapy. London: Royal Society of Medicine International Congress and Symposium: Series No. 74, 1985: 191-197.

32. The Canadian Clobazam Study Group for Childhood Epilepsy. Monotherapy clobazam vs. carbamazepine vs. phenytoin in childhood epilepsy: a double-blind randomized trial with 220 Canadian children. Can J Neurol Sci 1996; 23 (Suppl 1): S18.

33. Fisher $R$, Kälviäinen $R$, Tanganelli $P$, et al. Newer antiepileptic drugs as monotherapy: data on vigabatrin. Neurology 1996; 47(Suppl 1): S2-S5.
34. Kälviäinen R, Aikiä M, Saukkonen A et al. Vigabatrin vs carbamazepine monotherapy in patients newly diagnosed with epilepsy: a randomized controlled study. Arch Neurol 1995; 42: 989-996.

35. Tanganelli P, Regesta G. Vigabatrin vs. carbamazepine monotherapy in newly-diagnosed focal epilepsy: a randomized response conditional cross-over study. Epilepsy Res 1996; 25: 257-262.

36. Brodie MJ, Roy KB. One drug or two? A double-blind comparison of adjuvant vigabatrin and valproate in carbamazepine-resistant epilepsy. Epilepsia 1996; 37 (Suppl 5): S170.

37. Krauuss GL, Johnson MA, Miller NR. Vigabatrin-associated retinal cone system dysfunction. Electroretinogram and ophthalmologic findings. Neurology 1998; 50: 614-618.

38. Bergey GK, Morris HH, Rosenfeld W, et al. Gabapentin monotherapy. I: an 8-day, double-blind, dose-controlled, multicentre study in hospitalized patients with refractory complex partial or secondarily generalized seizures. Neurology 1997; 49: 739-745.

39. Beydoun A, Fisher J, Labar DR, et al. Gabapentin monotherapy. II: A 26-week, double-blind, dose-controlled, multicentre study of conversion from polytherapy in out-patients with refractory complex partial or secondarily generalized seizures. Neurology 1997; 49: 746-752.

40. Chadwick D, Anhut H, Murray G, et al. Gabapentin (GBP; Neurontin( $($ ) as monotherapy in newly-diagnosed patients with partial epilepsy: a fixed-dose comparison study vs. carbamazepine. Second European Congress of Epileptology, The Hague, September, 1996.

41. Sachdeo RC, Reife R, Pilar L, et al. Topiramate monotherapy for partial onset seizures. Epilepsia 1997; 38: 294-300. 